IVÁn Santiago GalarZa CaChiguango Candidato a doctor en Dirección de Empresas por la

Universidad de Valencia (España)

Profesor de Turismo y Gastronomía de la Universidad Técnica del Norte (UTN) (Ecuador)

[isgalarza@utn.edu.ec]

Carlos Aguinaga

Msc. Docente e investigador de la Universidad Técnica del Norte

Facultad de Ciencias Administrativas y Económicas, Carrera de Gastronomía (Ecuador)

[caaguinaga@utn.edu.ec]

PABlo LÓpez

Msc. Docente e investigador de la Universidad Técnica del Norte

Facultad de Ciencias Administrativas y Económicas, Carrera de Gastronomía (Ecuador)

[pxlopez@utn.edu.ec]

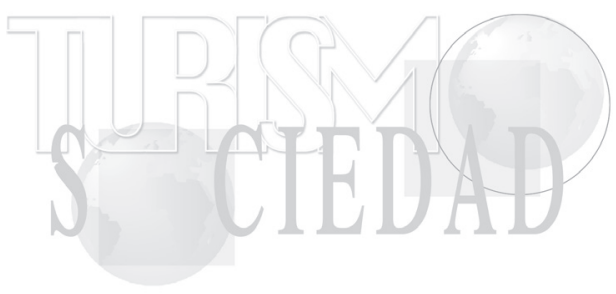

ROBERTO MOLINA Msc. Docente e investigador de la Universidad Técnica del Norte

Facultad de Ciencias Administrativas y Económicas, Carrera de Gastronomía (Ecuador)

[crmolina@utn.edu.ec]

Guadalupe Rosero Ortega Msc. Docente e investigadora de la Universidad Técnica del Norte

Facultad de Ciencias Administrativas y Económicas, Carrera de Gastronomía (Ecuador) [grosero@utn.edu.ec]

\section{LABORALES EN EL SECTOR DE LA RESTAURACIÓN: UN MARCO DE COMPETENCIAS CLAVE PARA SU GESTIÓN1}

\author{
LABOR COMPETENCES IN \\ THE RESTORATION SECTOR: \\ A FRAMEWORK OF KEY \\ COMPETENCES FOR ITS \\ MANAGEMENT
}

\section{Resumen}

El objetivo de este trabajo fue establecer un marco de competencias laborales para orientar la gestión de puestos clave en empresas de restauración. Tuvo un enfoque de dos dimensiones: competencias ocupacionales y personales. El diseño de la investigación fue de tipo fenomenográfico de corte trasversal; se llevó a cabo durante mayo y agosto de 2018 en la provincia de Imbabura (Ecuador). La unidad de análisis correspondió a restaurantes de primera y segunda categoría. La técnica de muestreo utilizada fue una muestra por criterio; el instrumento fue una entrevista semiestructurada. La pregunta de investigación fue la siguiente: ¿cuáles son las competencias laborales que las empresas del sector de la restauración requieren para una gestión eficiente de puestos clave de trabajo? Los resultados muestran que existe reducido personal con formación en competencias. Las competencias que tienen más significancia para procesos de gestión son las personales.

1 Para citar el artículo: Galarza, I., Aguinaga, C., López, P., Molina, R. y Rosero, G. (2020). Competencias laborales en el sector de la restauración: un marco de competencias clave para su gestión. Turismo y Sociedad, XXVII, pp. 161-181. DOI: https://doi.org/10.18601/01207555.n27.09

Fecha de recepción: 28 de mayo de 2019

Fecha de modificación: 8 de agosto de 2019

Fecha de aceptación: 21 de agosto de 2019 
Palabras clave: Competencias laborales, capacidades, marco de competencias, restauración, gastronomía.

\begin{abstract}
The objective of this work was to establish a framework of labor competences to guide the management of key positions in restoration companies. A two-dimensioned holistic approach was employed focusing on occupational and personal competences. The design of the research was a cross-sectional study in phenomenography. It was carried out during the months of May and August 2018, in the province of Imbabura, Ecuador. The analysis unit corresponded to first and second category restaurants. Sampling criteria was used, and consisted of a semi-structured interview. The research question was: What are the job competences that catering companies require for the efficient management of key jobs? The results show that there are few people with skills training; the competences seen as the most important for management processes are personal skills.
\end{abstract}

Keywords: Job skills, skills, skills framework, restoration, gastronomy.

\section{Introducción}

El marco de competencias laborales que orientan la gestión de puestos clave en empresas de restauración está basado en la aplicación y combinación de los conocimientos, las habilidades, las actitudes positivas y los valores que permiten llevar a cabo exitosamente una actividad laboral real.

El desempeño laboral está fundamentado en las competencias ocupacionales del talento humano, pero, sobre todo, en las competencias personales, las que en conjunto juegan un papel importante en el rendimiento organizacional.
Gersh (2016), en su estudio empírico sobre las percepciones de competencia en la industria culinaria, expresa:

Los estudios en el área de competencias en el sector de la restauración, más específicamente el aprendizaje basado en competencias en la industria de las artes culinarias, se encuentra[n] todavía en sus primeras etapas de desarrollo, las brechas son críticas, por lo que la participación de expertos de la industria, educadores y estudiantes son necesarios (sic) para orientar los currículos y las adquisiciones de competencias. (p. 33).

El presente estudio trata sobre las competencias clave que debe desarrollar el talento humano en el sector de la restauración. Existen reducidos trabajos con enfoque hacia la disciplina de los servicios; se han realizado estudios que describen las competencias funcionales que debe desarrollar el talento humano de empresas de servicios. Sin embargo, esta investigación aporta con un marco de competencias para siete puestos clave de trabajo en tres dimensiones fundamentales: cognitiva, funcional y social.

El enfoque de competencias considera que el desarrollo de competencias laborales desde una perspectiva holística contribuirá a fortalecer el sistema de gestión de recursos humanos: reclutamiento, selección, análisis de puestos, evaluación, formación y desarrollo de talentos. La formación, la capacitación y el desarrollo constituyen un proceso fundamental para el fortalecimiento y la adquisición de nuevas competencias personales y ocupaciones, además, son clave para hacer frente a los cambios internos y externos a los que se enfrentan las empresas de servicios.

A manera de justificación, se detallan algunas estadísticas de la industria de hoteles y restaurantes en Ecuador. En el año 2014, a nivel nacional fueron investigadas un total 
de 1.099 empresas de servicios de hotelería y restaurantes; de ese total, se identificó que el $32 \%$ se dedicaban a la actividad de hoteles y restaurantes; por su parte, el $68 \%$ restante correspondía a empresas de servicios en general, lo que demuestra la importancia de este sector en la economía del país (INEC, 2014).

Las empresas del servicio de alimentos y bebidas han sufrido un incremento significativo en los últimos años: los establecimientos registrados en el Ministerio de Turismo en 2012 fueron 13.261; en 2013, 14.057; en 2014, 15.741; en 2015, 17.325; y en el año 2016 sumaron 17.695. La contribución directa del turismo al PIB del Ecuador en 2017 fue del 2\%; el crecimiento de este sector en comparación con el año anterior fue del $14 \%$; y el número de empleados que registraba el turismo (alojamiento y servicio de comida) correspondía a 496.833 personas (Ministerio de Turismo, 2017).

Los datos reportados por el Ministerio de Turismo (2019) respecto a las actividades de turismo en Ecuador, con corte a 2018, reflejan un 2,8\% de contribución directa al PIB; 1.471.967 llegadas de extranjeros (se exceptúa Venezuela); ingreso por divisas estimadas por turismo receptor de $\$ 1.876,6$ millones; 463.320 empleados en las actividades de alojamiento y servicio de alimentos y bebidas; 24.720 establecimientos turísticos registrados en el Catastro. En cuanto al total de empleo en la industria del turismo a corte en el tercer trimestre de 2019, la cifra corresponde a 522.508 personas, lo que representa el 6,6\% del empleo nacional (Ministerio de Turismo, 2019).

Este trabajo está organizado de la siguiente forma: en el primer apartado se presenta la revisión de la literatura respecto a las competencias del sector de la restauración; en la segunda parte se encuentra la metodología; en el tercer bloque se exponen los resultados y la discusión; en la cuarta sección se hace la propuesta de un marco de competencias para puestos clave; por último, se presentan las conclusiones del estudio.

\section{Revisión de la literatura}

El término 'competencia' ha sido muy discutido. La raíz del enfoque de competencias se remonta a los trabajos de Katz en la década de los cincuenta del pasado siglo. Katz la definió como "una habilidad que puede desarrollarse, y no necesariamente innata, que se manifiesta en el rendimiento y no simplemente en el potencial".

El enfoque basado en la competencia se debe, básicamente, al desarrollo de las tecnologías, a la innovación de productos y procesos, al cambio demográfico, a la necesidad de reclutar recurso humano con habilidades universales, a la necesidad de capacitación y aprendizaje organizacional y, en especial, a la globalización (Le Deist y Winterton, 2005).

En el ámbito educativo, el término 'competencia' se introdujo en la década de los setenta como parte de la evaluación objetiva de los aprendizajes en la British Columbia University. Dicho enfoque consiste en que el diseño, el desarrollo y la evaluación curriculares se orientan a la probabilidad de movilizar un conjunto de recursos (saber, saber-hacer y saber-ser) para resolver una situación problema.

En cuanto a las competencias gerenciales específicamente con enfoque hacia las competencias laborales empresariales, fue David McClelland quien, en la década de los sesenta, llevó a cabo investigaciones y propuso una nueva variable para poder entender el concepto de motivación: desempeño-calidad. El autor considera el desempeño como la necesidad de logro (resultados cuantitativos), y la segunda, la calidad en el trabajo, como un resultado cualitativo. Siguiendo este 
enfoque, McClelland se plantea los posibles vínculos entre este tipo de necesidades y el éxito profesional, si se logran determinar los mecanismos o niveles de necesidades que mueven a los mejores empresarios.

McClelland demostró que ni los expedientes académicos ni las pruebas de inteligencia son suficientes por sí solos para adecuarse a los problemas de la vida cotidiana y, en consecuencia, al éxito profesional.

Las investigaciones de McClelland determinan cinco competencias laborales que se orientan al éxito de los gerentes (Gutiérrez, 2010).

- Competencias de logro y acción: motivación por el logro, preocupación por trabajar por competir para superar un estándar de excelencia.

- Competencias de influencia: deseo de producir un impacto o efecto sobre los demás.

- Competencias de dirección: gestión del recurso humano para establecer sinergia en la organización.

- Competencias cognitivas: pensamiento analítico, comprender situaciones y resolver problemas.

- Competencias de eficiencia personal: autoconfianza, autocontrol, compromiso.

Las competencias tienen características subyacentes en los seres humanos, es decir, son un aspecto profundo de las personas y perduran con el tiempo; además, son la causa de un desempeño exitoso, lo que significa que están relacionadas causalmente con un nivel de desempeño medido bajo cierto criterio.

McClelland identificó los siguientes dos grupos de características subyacentes: en el primer grupo están aquellas que integran la personalidad, es decir, los motivos, los rasgos y el autocontrol, que forman la parte oculta de las competencias; en el segundo grupo se encuentran los conocimientos y las habilidades, que son más visibles que las competencias del primer grupo (Lazzati, 2008).

\section{Definición de competencia}

El término 'competencia' proviene del vocablo latino competere, que significa pertenecer, incumbir, corresponder; es la habilidad y la idoneidad, es decir, ser competente para realizar algo. La palabra competencia empezó a emplearse en las ciencias administrativas, luego pasó a las ciencias jurídicas $\mathrm{y}$, posteriormente, a la educación (Morales, 2011).

Competencia se define como:

Un conjunto de conocimientos, habilidades $\mathrm{y}$ actitudes [relacionados] que afectan una parte importante del trabajo (un rol o responsabilidad), que se correlaciona con el desempeño en el trabajo, que puede medirse con respecto a estándares bien aceptados, y que puede ser mejorado a través de la capacitación y el desarrollo. (Lepsinger y De Lucía, 1999, p. 1).

En relación con la definición de competencia, Lazzati (2008, p. 179) expresa que "es la característica personal que está causalmente relacionada con un desempeño exitoso en un puesto de trabajo".

Martha Alles (2015b, p. 171) define la competencia como las "características de personalidad devenidas en comportamientos que generan un desempeño exitoso en un puesto de trabajo".

La Organización Internacional del Trabajo (OIT, 2012) define la competencia laboral como "una capacidad efectiva para llevar 
a cabo exitosamente una actividad laboral plenamente identificada. La competencia laboral no es una probabilidad de éxito en la ejecución de un trabajo; es una capacidad real y demostrada".

La definición de competencia en la que se basa el presente estudio corresponde a la propuesta por Arroyo (2012): las competencias son comportamientos observables que se pueden demostrar por medio de la aplicación de conocimientos, habilidades, actitudes y valores, los cuales permiten llevar a cabo exitosamente un actividad laboral real.

El conocimiento, visto desde la epistemología tradicional, es una creencia verdadera justificada (Nonaka, 1994, p. 15). La habilidad es la capacidad para hacer, alude a la acción de llevar a la práctica el conocimiento. La actitud es la predisposición de una persona hacia un objeto, se refiere al comportamiento que emplea una persona para realizar algo. Los valores son manifestaciones mentales profundas de las personas que orientan y determinan su conducta.

En este trabajo, el enfoque de competencias laborales es holístico y adopta los conceptos respecto a la clasificación de competencias de Echeverría (2002) expuestos en la tabla 1, así como las dimensiones ocupacionales y personales de Le Deist y Winterton, presentadas en la figura 1.
Figura 1. Dimensiones de la competencia

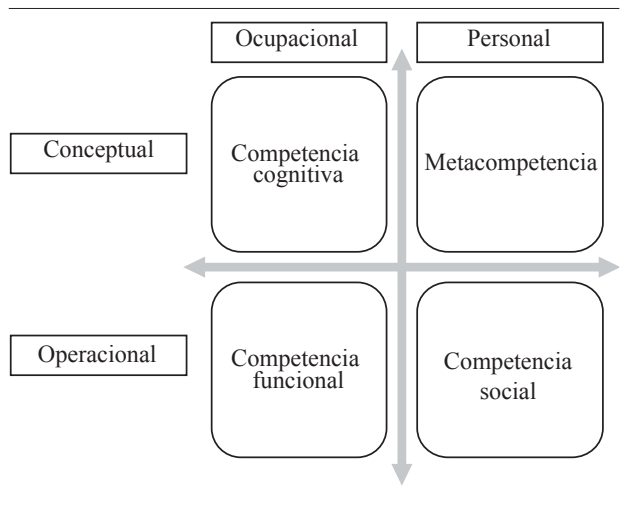

Fuente: Le Deist y Winterton (2005).

Las dimensiones de las competencias son descritas por Baradarani y Kilic (2017) de la siguiente forma:

Competencias cognitivas de conocimiento: aquellas relacionadas con el conocimiento conceptual del individuo.

Competencias funcionales: relacionadas con las habilidades técnicas de las personas en sus puestos de trabajo.

Competencias sociales: actitudes y comportamientos interpersonales para interactuar efectivamente con los demás.

Metacompetencias: motivos y rasgos de orden superior relacionados con la capacidad de comprenderse y comprender la situación

Tabla 1. Clasificación de las competencias por acciones

\begin{tabular}{|c|c|c|c|}
\hline Competencia técnica & Competencia metodológica & Competencia participativa & Competencia personal \\
\hline Saber & Saber hacer & Saber estar & Saber ser \\
\hline $\begin{array}{l}\text { Dominar los conocimien- } \\
\text { tos necesarios vinculados } \\
\text { a la actividad laboral }\end{array}$ & $\begin{array}{l}\text { Aplicar los conocimientos } \\
\text { y las técnicas a situaciones } \\
\text { concretas y desarrollar estas } \\
\text { experiencias en nuevas situa- } \\
\text { ciones laborales }\end{array}$ & $\begin{array}{l}\text { Comportarse en un grupo } \\
\text { de manera colaboradora y } \\
\text { comunicarse de forma ade- } \\
\text { cuada en el ámbito social }\end{array}$ & $\begin{array}{l}\text { Tomar decisiones, asumir } \\
\text { responsabilidades y } \\
\text { actuar de acuerdo con las } \\
\text { convicciones personales }\end{array}$ \\
\hline
\end{tabular}

Fuente: Adaptado de Echeverría (2002). 
Tabla 2. Países del mundo y organismos que certifican competencias laborales

\begin{tabular}{|c|c|c|}
\hline País & Año & Organismos impulsores y encargados de la certificación de competencias laborales \\
\hline Francia & $\begin{array}{c}\text { Década } \\
\text { de los } 70\end{array}$ & $\begin{array}{l}\text { Sistema de Formación Profesional, a cargo del Estado } \\
\text { Centres Interinstitutionnels de Bilans de Compétences (CIBC) }\end{array}$ \\
\hline $\begin{array}{l}\text { República } \\
\text { Dominicana }\end{array}$ & 1980 & Instituto Nacional de Formación Técnico Profesional (INFOTEP) \\
\hline Honduras & 1983 & Centro Asesor para el Desarrollo de los Recursos Humanos (CADERH) \\
\hline Gran Bretaña & 1986 & National Council for Vocational Qualifications (NCVQ) \\
\hline Australia & 1987 & $\begin{array}{l}\text { Australian National Training Authority (ANTA) } \\
\text { Australian Qualifications Framework (AQF) }\end{array}$ \\
\hline Brasil & 1990 & Red Nacional de Certificaciones \\
\hline Nicaragua & 1991 & Instituto Nacional Tecnológico (INATEC) \\
\hline Paraguay & 1992 & Cámara Paraguaya de la Construcción \\
\hline El Salvador & 1993 & Instituto Salvadoreño de Formación Profesional (INSAFORP) \\
\hline México & 1995 & $\begin{array}{l}\text { Consejo de Normalización de Competencias Laborales (CONOCER), Proyecto de Mo- } \\
\text { dernización de la Educación Técnica y Capacitación (PMETyC) }\end{array}$ \\
\hline Bolivia & 1996 & Instituto Nacional de Formación y Capacitación Laboral (INFOCAL) \\
\hline Uruguay & 1997 & Dirección Nacional de Empleo de Uruguay, BID, Fondo Mundial de Inversiones \\
\hline Guatemala & 1998 & Instituto Técnico de Capacitación y Productividad (INTECAP) \\
\hline España & 1998 & Instituto Nacional de Cualificaciones (INCUAL) \\
\hline Chile & 1999 & Fundación Chile, sector público, sector productivo \\
\hline Japón & 2002 & $\begin{array}{l}\text { Sistema Global de Calificaciones Profesionales (Comprehensive Vocational Qualifica- } \\
\text { tions System) } \\
\text { Sistema de Calificación (Qualifications System), incluye certificaciones otorgadas por } \\
\text { empresas privadas. }\end{array}$ \\
\hline Ecuador & 2015 & $\begin{array}{l}\text { Secretaría Técnica del Sistema Nacional de Cualificaciones y Capacitación Profesio- } \\
\text { nal (SETEC) }\end{array}$ \\
\hline Alemania & & Instituto Federal para la Formación Profesional (BIBB) \\
\hline
\end{tabular}

Fuente: Elaboración propia tomando como base a Schkolnik, Araos y Machado (2005).

Tabla 3. Perfiles de puestos del sector hotelería y restauración que se certifican en Ecuador, México y España

\begin{tabular}{|l|l|l|}
\hline \multicolumn{1}{|c|}{ Ecuador } & \multicolumn{1}{c|}{ México } & \multicolumn{1}{c|}{ España } \\
\hline Preparaciones culinarias de partida & No aplica & Operaciones básicas de cocina \\
\hline $\begin{array}{l}\text { Preparaciones y servicio de bebidas en el bar o } \\
\text { llamado barman }\end{array}$ & Barman & Servicios de bar y cafetería \\
\hline Dirección de meseros & No aplica & \\
\hline Mesero polivalente & No aplica & Servicios de restaurante \\
\hline
\end{tabular}




\begin{tabular}{|c|c|c|}
\hline Ecuador & México & España \\
\hline Preparaciones gastronómicas de cocina & Cocineros & Cocina \\
\hline Preparaciones panaderas y pasteleras & $\begin{array}{l}\text { Panaderos, pasteleros y } \\
\text { confiteros }\end{array}$ & Dirección y producción de pastelería \\
\hline Gestión culinaria o denominado chef de cocina & Jefe de cocina (restaurante) & Dirección y producción de cocina \\
\hline Ayudante de bar tender & Cantineros & \\
\hline Ayudante de pastelería & No aplica & Operaciones básicas de pastelería \\
\hline Hostess & No aplica & No aplica \\
\hline Técnicas de arte culinario ecuatoriano & No aplica & No aplica \\
\hline Ayudante de cocina & Ayudante de cocinero & No aplica \\
\hline Ayudante de mesero & $\begin{array}{l}\text { Auxiliares de servicios de } \\
\text { alimentos y bebidas }\end{array}$ & No aplica \\
\hline Administrador de restaurantes & No aplica & Dirección en restauración \\
\hline Capitán de meseros & No aplica & No aplica \\
\hline Servicio de catering & No aplica & Operaciones básicas de catering \\
\hline Parrillero & No aplica & No aplica \\
\hline Maître & Capitán de restaurante & No aplica \\
\hline Carnicería y charcutería & No aplica & No aplica \\
\hline Servicio de vinos y licores & No aplica & Sumillería \\
\hline
\end{tabular}

Fuente: Instituto Nacional de Cualificaciones (2018), Secretaría de Educación Pública de México (2018) y SETEC Ecuador (2018).

de su entorno para adaptar y aplicar competencias o adquirir nuevas, según sea el escenario.

\section{Metodología}

El diseño de la investigación fue de tipo fenomenográfico. A partir de este diseño se intenta comprender la perspectiva cognitivoconceptual, es decir, la realidad que tiene el empresario de la restauración respecto a las competencias de puestos de trabajo y su gestión. La investigación fue de carácter trasversal y se llevó a cabo durante mayo y agosto de 2018 en la provincia de Imbabura (Ecuador). La unidad de análisis correspondió a restaurantes clasificados como de primera y segunda categoría.
La técnica de muestreo utilizada fue una muestra por criterio; estos fueron los criterios de base: restaurantes registrados en la base de datos del Ministerio de Turismo del Ecuador en 2018, que se encontraran operando por más de 5 años en el mercado y cuya categoría correspondiera a los cuartiles primero y segundo. En total, los establecimientos que reunieron este criterio, y a los que se aplicó el instrumento, fueron 12 restaurantes en Otavalo, 27 en Ibarra, 11 en Cotacachi, 5 en Urcuquí, 17 en Antonio Ante y 4 en Ambuquí, para un total de 76 establecimientos.

La técnica usada para la recolección de datos fue una entrevista semiestructurada, la cual fue aplicada a los directores o administradores de los restaurantes. Para la elaboración del instrumento se usó información 
de los perfiles de la Secretaría Técnica del Sistema Nacional de Cualificaciones y Capacitación Profesional SETEC Ecuador; en esta Secretaría, se identificó que se certifican varios puestos de trabajo en el sector del turismo y la alimentación; estos perfiles se pueden observar en la tabla 3 .

Así mismo, para la conformación del instrumento se usaron los resultados de aprendizaje de la carrera de Gastronomía de la UTN, las normas del INEN Ecuador, el enfoque de certificaciones de profesionalización en hotelería y restauración de España y las dos dimensiones de competencia: ocupacional y personal (Le Deist y Winterton, 2005).

Dado que el objetivo era investigar las competencias de los puestos clave de restaurantes en concordancia con los puestos declarados como principales para operar un restaurante, se llegó a determinar que estos corresponden a chef de partida, ayudante de cocina, dirección de meseros, chef pastelero, repostero y mesero. Una vez determinados los puestos clave que se iban a investigar, se procedió a diseñar el instrumento. El desarrollo de esta actividad estuvo a cargo de cinco profesores especialistas en diferentes áreas de la gastronomía y la hotelería de la carrera de la UTN, quienes, en conjunto con estudiantes de la propia carrera, procedieron a revisar la comprensión de la entrevista.

El instrumento fue elaborado con base en un enfoque de competencias e incluía preguntas relacionadas con las actividades que se realizan en los puestos clave de los restaurantes. Con el objetivo de otorgar fiabilidad a la información que se obtendría, se decidió incluir en el cuestionario ítems asociados con los tipos de evaluación de desempeño y posibles riesgos laborales vinculados a estos. Al respecto, Chiavenato (2009, p. 221) señala:

En una definición de puestos de trabajo se identifica, describe y define un puesto en términos de obligaciones, responsabilidades, condiciones de trabajo, se relacionan los conocimientos, [las] habilidades y las capacidades que se necesitan para el desempeño satisfactorio de puestos de trabajo.

Una vez discutidos estos aspectos, se realizaron los ajustes y se concluyó con un solo instrumento.

Las actividades declaradas en los puestos de trabajo fueron relacionadas con las competencias laborales que se certifican en Ecuador, España y México (ver tabla 3); con esta base se construyó el marco de competencias en sus dos dimensiones: ocupacional y personal.

Del total de las empresas de la muestra, las que efectivamente contestaron la entrevista fueron 33, ello representa el 43,42\% de la muestra. Cuando se aplicó el instrumento, se pudo evidenciar que cerca del $10 \%$ de las empresas registradas en el catastro del Ministerio de Turismo del Ecuador ya no operaban. Otro factor adicional a la negativa para participar en este tipo de proyectos se debió a que los directivos de las empresas asocian la información que brindan con sistemas de control para sus organizaciones. Un elemento motivador que se incluyó al momento de la entrevista fue consultar a los administradores y propietarios de los restaurantes acerca de su disposición a participar en un proyecto de desarrollo de competencias en la modalidad virtual; este trabajo será objeto de otro estudio.

\section{Resultados y discusión}

La entrevista semiestructurada aplicada a los 33 restaurantes de primera y segunda categoría de la provincia de Imbabura (Ecuador) permitió obtener los siguientes resultados:

El número de personas que trabajan en los restaurantes específicamente en el área de alimentos y bebidas es de 2 a 5 trabajadores, 
lo que corresponde al $33 \%$; de 6 a 8 personas (39\%); entre 9 y 12 personas $(15 \%)$; y más de 4 trabajadores (12\%).

A nivel mundial, se concibe que las mipymes conforman una porción muy importante de la actividad económica — que en promedio corresponden al $90 \%$ de las empresas - y que, a su vez, generan más de la mitad del empleo que ofrece la actividad económica empresarial, con una participación en el producto interno bruto (PIB) que se ubica en un promedio del $50 \%$, dependiendo del tipo de producción.

En Ecuador, la realidad es muy similar. De acuerdo con información obtenida por el Instituto Nacional de Estadísticas y Censos (INEC), en el año 2011, la participación de las mipymes representaba el $84,3 \%$ del total de los establecimientos, de modo que este tipo de empresas ocupó al 37,7\% de los trabajadores y generó un aporte del $13 \%$ al PIB (Romero, 2006; Yance, Solís, Burgos y Hermida, 2017).

En cuanto a los puestos de trabajo que existen en las empresas entrevistadas, 18 restaurantes indicaron que cuentan con el puesto de chef de partida, la misma cantidad dijo tener el puesto de ayudante de cocina, 13 declararon el puesto de mesero, 9 establecimientos no identificaron ningún puesto de trabajo, 7 mencionaron la dirección de meseros e igual número citaron el puesto de chef pastelero, 4 cuentan con el puesto de panadero y 3 establecimientos reportaron el puesto de chef repostero. Estos mismos puestos de trabajo son los que se certifican en otros países, como España y México (ver tabla 3).

La disposición a participar en programas de desarrollo de competencias en los que se incluya la modalidad virtual como mecanismo de desarrollo de competencias refleja una respuesta positiva del $82 \%$ versus una negativa del $18 \%$. Los puestos que se consideran clave para la operación de una empresa restaurantera y que estarían dispuestos a participar en programas de capacitación en la modalidad virtual son, en orden de importancia, chef de partida, ayudante de cocina, dirección de meseros, chef pastelero, repostero y mesero. Al respecto, el estudio de Yap, Jung y Kisseleff (2015, p. 8) realizado en instituciones de educación superior privada en Suiza destaca:

En general[,] la modalidad e-learning era beneficiosa para el crecimiento y [la] madurez personal de los estudiantes de hotelería, les ayuda a ampliar los horizontes y [a] prepararlos para un trabajo en la industria; sin embargo[,] hay que considerar que esta modalidad necesita programas de capacitación y entrenamiento continuo de los participantes y docentes para mejorar las funcionalidades y [los] usos de la plataforma; algunas de las desventajas en el uso de las plataformas e-learning fueron el tiempo dedicado a la configuración inicial de la plataforma, la carga de archivos interactivos y la actualización de versiones nuevas.

Las actividades principales que se realizan en los puestos clave reflejan la siguiente información:

Puesto preparaciones gastronómicas de cocina (P1). Las actividades declaradas son realizar el mise en place, limpiar las áreas de la cocina y elaborar preparaciones básicas de alimentos.

Puesto dirección de meseros (P2). Las actividades principales corresponden a la elaboración y estandarización de procesos del servicio acordes con los requerimientos del establecimiento, también a controlar al equipo a su cargo y dirigir el área de trabajo. 
Puesto chef de partida (P3). Las actividades que se realizan en este puesto de trabajo son la dirección de su área de trabajo y la ejecución de procesos de requisición de materias primas y de órdenes de trabajo encaminadas a la elaboración de productos. Otras actividades son la preparación de platillos, la elaboración de menús, la revisión de la limpieza de las áreas de trabajo, la planificación de cartas y menús, la gestión de compras y el control del equipo de trabajo.

Puesto pastelero (P4). Las actividades declaradas comprenden preparaciones culinarias relativas a la pastelería-repostería, elaboración de recetas estándar e innovación de postres.

Puesto repostero (P5). Se destacan la elaboración de postres del menú, la planificación de compras y la estandarización de recetas.

Puesto panadero (P6). Las actividades indicadas son organización del puesto de trabajo, actividades de mise en place para la elaboración de variedad de panes nacionales y mundiales, y elaboración de postres en general.

Puesto mesero (P7). Las actividades prioritarias que se desatacan son atención al cliente, inventario de mantelería, inventario de vajilla, montaje del área de comedor, servicio de restaurante, aseo general del área del comedor, organización del servicio; además, es el responsable del estado de los materiales para el servicio de alimentos.

En relación con las competencias personales, la mayoría de los entrevistados consideran estas competencias como elementos imprescindibles en puestos de trabajo para la gestión del talento humano. En orden de importancia y valoración, estas competencias corresponden a compromiso con la organización, responsabilidad, ética, integridad, creatividad, respeto, disciplina, flexibilidad, adaptación, prudencia y temple.

Con respecto al número de empleados que cuentan con certificados de competencias laborales, los datos reflejan que, de los 33 restaurantes (área del servicio de alimentos y bebidas), 22 tienen certificaciones en competencias; en las empresas de 1 a 5 empleados existen 8 personas con certificación en competencias; en empresas de 6 a 10 personas hay 2 personas certificadas; $y$ en aquellas con más de 10 colaboradores solo 1 persona tiene este tipo de certificados.

De acuerdo con la SETEC Ecuador (Secretaría Técnica del Sistema Nacional de Cualificaciones Profesionales), hasta diciembre de 2017, por medio de los Organismos Evaluadores de la Conformidad (OEC) reconocidos ante la SETEC, se ha certificado a 45.483 personas, de las cuales 2.649 corresponden al sector turismo, en el que se incluyen los establecimientos de alimentos y bebidas. El Catálogo Nacional de Cualificaciones (CNC) inició en 2017 con 571 perfiles, y a diciembre de 2017 se publicaron 162 perfiles en el CNC. Actualmente, la SETEC dispone de un total de 733 perfiles incluidos en el Catálogo para los 22 sectores definidos por las comisiones sectoriales del Consejo Nacional del Trabajo, de los cuales 75 corresponden al sector turismo y alimentación (SETEC Ecuador, 2017).

E1 $67 \%$ de los entrevistados consideran que la certificación de competencias es imprescindible para la gestión de puestos de trabajo, versus un $33 \%$ que no la consideran así. El $70 \%$ de los entrevistados declararon realizar evaluaciones de desempeño, en cambio, un $30 \%$ no lo hace. Los métodos de evaluación del desempeño que usan las empresas son lista de verificación de actividades (16 empresas); evaluaciones por medio del superior, del jefe o son autoevaluaciones 
(12 empresas); y lista de verificación de comportamientos ( 9 empresas). Una empresa declaró usar otros métodos de evaluación, y 13 manifestaron no realizar ningún tipo de evaluación.

Entre los beneficios que puede aportar a los trabajadores el contar con personal certificado en competencias en la empresa, se incluyen el reconocimiento de orden social por el ejercicio de su trabajo, el poder adquirir y desarrollar nuevas habilidades acordes con los cambios sufridos por los avances organizacionales o tecnológicos, la transferencia de estas nuevas habilidades al entorno empresarial y otras actividades laborales. De igual manera, hace posible tener una idea de cómo se encuentra su situación con respecto al mercado laboral, lo que le permitirá al trabajador realizar una búsqueda más adecuada de empleo. Por último, les generará mejores oportunidades de promociones y vinculaciones laborales.

Para el sector productivo, los beneficios consisten en lograr una mayor armonía dentro de la organización al contar con personal con estándares profesionales; permitir un desarrollo de la calidad en las organizaciones; mejorar los procesos de contratación, ya que favorece tener una idea más acertada de las necesidades laborales de la empresa y de capacitación en esta; hay una disminución en los costos al momento de formular los planes de capacitación; por último, se promueven la productividad y la competitividad en la empresa mediante el mejoramiento continuo. Y a los oferentes de formación les permite estructurar y regular la oferta de sus productos en cuanto a la calidad, pertinencia y oportunidad de sus cursos de capacitación o formación (SENA, 2011).

Los tipos de riesgos laborales identificados en los puestos clave de trabajo son físicos (identificados por 21 empresas), mecánicos (17 empresas), ergonómicos (16 empresas) y psicosociales (12 empresas); 11 organizaciones declararon que los puestos de trabajo no tienen riesgos asociados. E1 55\% de los entrevistados enunciaron que se han producido accidentes laborales en sus empresas, por su parte, el $45 \%$ manifestaron que no se ha generado ningún accidente.

Dado el índice de riesgos (55\%), es menester destacar que las normativas y los decretos de salud ocupacional (Ecuador) mencionan que "es un derecho de las empresas otorgar las condiciones y [los] ambientes laborales adecuados, para el óptimo y libre desempeño de funciones propias del puesto de trabajo, garantizando la salud, integridad, seguridad, higiene y bienestar". En tal sentido, es obligación de la empresa diseñar un plan de mantenimiento preventivo y correctivo de equipos, maquinarias y del propio lugar de trabajo para garantizar el desarrollo de las actividades con seguridad.

Cuando se han identificado los riesgos o estos, a su vez, se han materializado en las empresas, es obligación del empleador elaborar planes integrales de prevención de riesgos que comprendan los siguientes puntos: combatir los riesgos desde su origen o transmisión, diseñar estrategias para la puesta en práctica de medidas de prevención y fomentar la adaptación del trabajo y de los puestos de trabajo a las capacidades de los trabajadores, habida cuenta de su estado de salud física y mental.

\section{Marco genérico holístico de competencias laborales de puestos clave para empresas de restauración}

El objetivo de este marco de competencias para empresas pequeñas y medianas de restauración está basado en las actividades principales que se realizan en los puestos clave de las empresas. Toma como bases las definiciones de competencias laborales de la Secretaría de Cualificación y Profesionalización 
SETEC Ecuador, los conceptos respecto a los comportamientos más utilizados en la gestión por competencias de Alles (2015a), el Diccionario de competencias de la Universidad de Santiago de Chile, el enfoque holístico de competencias ocupacionales personales de Le Deist y Winterton (2005) y la discusión de una mesa de trabajo con cinco especialistas en el área de servicios de hotelería y restaurantes de la carrera de Gastronomía de la UTN.

\section{Competencias ocupacionales}

Tabla 4. Dimensiones de las competencias cognitiva $\mathrm{y}$ funcional. Puesto 1: cocinero

\begin{tabular}{|c|c|}
\hline Competencias cognitivas & Competencias funcionales \\
\hline $\begin{array}{l}\text { P1. Cc1. Conoce los riesgos laborales de su puesto de } \\
\text { trabajo de acuerdo con las normas de seguridad y ries- } \\
\text { gos laborales. }\end{array}$ & $\begin{array}{l}\text { P1. CF1. Comprueba y verifica el funcionamiento del } \\
\text { equipo pesado (mesas, cámaras, freidoras, cortadoras, } \\
\text { hornos, otros) y del equipo liviano (cuchillos, tablas, } \\
\text { varillas, moldes, otros). }\end{array}$ \\
\hline $\begin{array}{l}\text { P1. CC2. Conoce el manejo de la materia prima y el } \\
\text { registro de inventarios según los sistemas FIFO y las } \\
\text { normas de seguridad alimentaria. }\end{array}$ & $\begin{array}{l}\text { P1. CF2. Realiza la requisición de productos de materia } \\
\text { prima registrándolos en el software específico para ese } \\
\text { fin. }\end{array}$ \\
\hline $\begin{array}{l}\text { P1. CC3. Conoce los diferentes tipos de cortes de verdu- } \\
\text { ras y hortalizas (brunoise, juliana, batonnet, otros), así } \\
\text { como distintos cortes de carne que se usan en hotelería, } \\
\text { restauración y gastronomía según las técnicas gastronó- } \\
\text { micas actuales. }\end{array}$ & $\begin{array}{l}\text { P1. CF3. Realiza el corte de verduras, frutas y productos } \\
\text { cárnicos de acuerdo con técnicas culinarias y según la } \\
\text { cantidad requerida en la receta estándar. }\end{array}$ \\
\hline $\begin{array}{l}\text { P1. cc4. Reconoce la documentación específica (receta } \\
\text { estándar) que contiene las normas establecidas para un } \\
\text { determinado producto, en las que se incluyen las técni- } \\
\text { cas y los procedimientos aplicados a la preparación. }\end{array}$ & $\begin{array}{l}\text { P1. CF4. Realiza el mise en place aplicando técnicas co- } \\
\text { mo batido, empanado, mezclado, blanqueado, sazonado, } \\
\text { marinado, entre otras, de acuerdo con las políticas del } \\
\text { establecimiento. }\end{array}$ \\
\hline $\begin{array}{l}\text { P1. CC5. Conoce distintas técnicas de cocción en la } \\
\text { preparación de las recetas y que involucran la modifica- } \\
\text { ción de los alimentos mediante la aplicación de horne- } \\
\text { ado, frito, salteado, confitado, entre otras. }\end{array}$ & $\begin{array}{l}\text { P1. CF5. Prepara los alimentos de acuerdo con las técni- } \\
\text { cas y los métodos de cocción de la receta estándar. }\end{array}$ \\
\hline $\begin{array}{l}\text { P1. CC6. Identifica las técnicas de montaje de platos } \\
\text { según las diferentes tendencias presentes en el mercado } \\
\text { (tradicional, no tradicional, disperso, estructurado, etc.), } \\
\text { teniendo en cuenta la cantidad de proteínas, carbohidra- } \\
\text { tos y vegetales que se incluirán en el plato. }\end{array}$ & $\begin{array}{l}\text { P1. CF6. Verifica que la higiene de la vajilla, la tempera- } \\
\text { tura y el montaje de la oferta gastronómica correspon- } \\
\text { dan al diseño establecido. }\end{array}$ \\
\hline $\begin{array}{l}\text { P1. CC7. Identifica las normas de seguridad alimentaria } \\
\text { de acuerdo con las normas legales, sanitarias y del ám- } \\
\text { bito laboral en el que desempeña sus funciones. }\end{array}$ & $\begin{array}{l}\text { P1. CF7. Higieniza la estación de trabajo (equipos y } \\
\text { utensilios) con base en los protocolos establecidos. }\end{array}$ \\
\hline
\end{tabular}

$\mathrm{P}=$ Puesto

$\mathrm{CC}=$ Competencia cognitiva

$\mathrm{CF}=$ Competencia funcional

Fuente: Elaboración propia (2019). 
Tabla 5. Dimensiones de las competencias cognitiva y funcional.

Puesto 2: dirección de meseros

\begin{tabular}{|c|c|}
\hline Competencias cognitivas & Competencias funcionales \\
\hline $\begin{array}{l}\text { P2. CC1. Describe el protocolo de servicio y etiqueta se- } \\
\text { gún las normas de etiqueta y protocolo establecidas por } \\
\text { el uso o por el lugar donde va a ejercer sus labores. }\end{array}$ & $\begin{array}{l}\text { P2. CF1. Actúa de manera adecuada frente a los clientes, } \\
\text { siguiendo el protocolo de servicio y atención en la mesa } \\
\text { establecido. }\end{array}$ \\
\hline $\begin{array}{l}\text { P2. CC2. Identifica el proceso de marcar y desbarazar } \\
\text { mesas según las normas de protocolo y etiqueta recono- } \\
\text { cidas o de acuerdo con el protocolo donde va a ejercer } \\
\text { sus labores. }\end{array}$ & $\begin{array}{l}\text { P2. CF2. Verifica la mantelería, los platos y el petit me- } \\
\text { nage de acuerdo con los requerimientos establecidos. }\end{array}$ \\
\hline $\begin{array}{l}\text { P2. CC3. Identifica bases de cocina que incluyen conoci- } \\
\text { mientos básicos de ingredientes y diferentes prepara- } \\
\text { ciones gastronómicas de acuerdo con las técnicas y la } \\
\text { cultura local e internacional. }\end{array}$ & $\begin{array}{l}\text { P2. CF3. Sugiere al cliente las ofertas del día o bebidas, } \\
\text { de ser el caso, de acuerdo con su preferencia. }\end{array}$ \\
\hline $\begin{array}{l}\text { P2. CC4. Define las técnicas de atención al cliente según } \\
\text { el protocolo de servicio establecido. }\end{array}$ & $\begin{array}{l}\text { P2. CF4. Resuelve las inquietudes del cliente de acuerdo } \\
\text { con sus necesidades. }\end{array}$ \\
\hline $\begin{array}{l}\text { P2. CC5. Reconoce la comunicación efectiva para la to- } \\
\text { ma de pedidos de acuerdo con las normas de protocolo } \\
\text { y etiqueta. }\end{array}$ & $\begin{array}{l}\text { P2. CF5. Toma el pedido y anota en la comanda los dife- } \\
\text { rentes tipos de alimentos y/o bebidas. }\end{array}$ \\
\hline $\begin{array}{l}\text { P2. CC6. Memoriza el correcto montaje de platos, que } \\
\text { incluye la forma en que los diferentes alimentos se dis- } \\
\text { tribuyen dentro según la estética establecida. }\end{array}$ & $\begin{array}{l}\text { P2. CF6. Verifica el despacho de platos y bebidas de } \\
\text { acuerdo con la comanda realizada y los requerimientos } \\
\text { adicionales en algunos casos. }\end{array}$ \\
\hline $\begin{array}{l}\text { P2. CC7. Define la mejora continua según los principios } \\
\text { de la calidad, lo que implica un seguimiento de los pro- } \\
\text { cesos para buscar mejoras constantes en estos. }\end{array}$ & $\begin{array}{l}\text { P2. CF7. Evalúa el servicio con respecto al cliente, y pa- } \\
\text { ra ello considera opiniones y sugerencias de los clientes. }\end{array}$ \\
\hline
\end{tabular}

Fuente: Elaboración propia (2019).

Tabla 6. Dimensiones de las competencias cognitiva y funcional.

Puesto 3: chef de cocina

\begin{tabular}{|l|l|}
\hline \multicolumn{1}{|c|}{ Competencias cognitivas } & \multicolumn{1}{c|}{ Competencias funcionales } \\
\hline $\begin{array}{l}\text { P3. CC1. Clasifica la merma y el desperdicio de acuerdo } \\
\text { con las normas y los controles para la reducción de } \\
\text { costos. }\end{array}$ & $\begin{array}{l}\text { P3. CF1. Realiza y planifica el control de materias pri- } \\
\text { mas como mermas y desperdicios de alimentos. }\end{array}$ \\
\hline $\begin{array}{l}\text { P3. CC2. Reconoce las codificaciones y los sistemas de } \\
\text { comandas según la carta del establecimiento, de modo } \\
\text { que permite su producción de manera eficiente. }\end{array}$ & $\begin{array}{l}\text { P3. CF2. Recibe comanda de servicios para la prepara- } \\
\text { ción de alimentos de acuerdo con la oferta sugerida. }\end{array}$ \\
\hline $\begin{array}{l}\text { P3. CC3. Reconoce estándares para el montaje de platos } \\
\text { según la estética establecida. }\end{array}$ & $\begin{array}{l}\text { P3. CF3. Direcciona y controla el resultado de los mon- } \\
\text { tajes de acuerdo con las políticas establecidas. }\end{array}$ \\
\hline $\begin{array}{l}\text { P3. CC4. Escribe menús y cartas de acuerdo con la oferta } \\
\text { gastronómica del establecimiento. }\end{array}$ & $\begin{array}{l}\text { P3. CF4. Diseña y presenta sugerencias para la construc- } \\
\text { ción de cartas y menús de acuerdo con las exigencias } \\
\text { del mercado. }\end{array}$ \\
\hline $\begin{array}{l}\text { P3. CC5. Reconoce la importancia de la gestión de } \\
\text { recursos humanos para impulsar el potencial del talento } \\
\text { humano de acuerdo con las normas fijadas por el esta- } \\
\text { blecimiento. }\end{array}$ & $\begin{array}{l}\text { P3. CF5. Administra los recursos (equipos y personal del } \\
\text { servicio) de acuerdo con los estándares de la empresa. }\end{array}$ \\
\hline
\end{tabular}




\begin{tabular}{|l|l|}
\hline \multicolumn{1}{|c|}{ Competencias cognitivas } & \multicolumn{1}{c|}{ Competencias funcionales } \\
\hline $\begin{array}{l}\text { P3. CC6. Identifica las técnicas de procesos de acuerdo } \\
\text { con los sistemas de calidad (normas ISO, HACCP, EFQM). }\end{array}$ & $\begin{array}{l}\text { P3. CF6. Controla los procesos de producción y optimiza } \\
\text { tiempos, stock, equipos y recursos en la transformación } \\
\text { de las materias primas. }\end{array}$ \\
\hline $\begin{array}{l}\text { P3. CC7. Identifica las técnicas de control de temperatu- } \\
\text { ras y conservación de los alimentos de acuerdo con las } \\
\text { normas de seguridad alimentaria, los sistemas de puntos } \\
\text { críticos de control y las normas de calidad. }\end{array}$ & $\begin{array}{l}\text { P3. CF7. Aplica técnicas de conservación y cocción de } \\
\text { los alimentos, para lo cual toma en consideración las } \\
\text { normas de seguridad alimentarias. }\end{array}$ \\
\hline
\end{tabular}

Fuente: Elaboración propia (2019).

Tabla 7. Dimensiones de las competencias cognitiva y funcional. Puesto 4: pastelero

\begin{tabular}{|c|c|}
\hline Competencias cognitivas & Competencias funcionales \\
\hline $\begin{array}{l}\text { P4. CC1. Conoce sistemas, métodos y documentos que } \\
\text { se aplican para la gestión de inventarios de acuerdo con } \\
\text { los sistemas de rotación, como FIFO, LIFO Y PROMEDIO. }\end{array}$ & $\begin{array}{l}\text { P4. CF1. Organiza la rotación de materias primas, pro- } \\
\text { ductos en proceso y producto terminado. }\end{array}$ \\
\hline $\begin{array}{l}\text { P4. CC2. Reconoce, basado en la receta estándar y el } \\
\text { escandallo, los procesos de producción acordes con las } \\
\text { políticas de la empresa. }\end{array}$ & $\begin{array}{l}\text { P4. CF2. Prepara y arregla el área de trabajo para la } \\
\text { puesta a punto de las materias primas (mise en place). }\end{array}$ \\
\hline $\begin{array}{l}\text { P4. CC3. Identifica las normas de seguridad alimentaria } \\
\text { según los parámetros de higiene personal y los métodos } \\
\text { físicos o químicos para la limpieza de superficies, equi- } \\
\text { pos y utensilios. }\end{array}$ & $\begin{array}{l}\text { P4. CF3. Coordina de forma permanente la limpieza, } \\
\text { higiene y seguridad antes, durante y luego de la produc- } \\
\text { ción de alimentos. }\end{array}$ \\
\hline $\begin{array}{l}\text { P4. cc4. Identifica técnicas de pastelería básicas y de } \\
\text { complejidad intermedia de acuerdo con las bases de la } \\
\text { cocina regional, internacional y de vanguardia. }\end{array}$ & $\begin{array}{l}\text { P4. CF4. Prepara y coordina la elaboración de rellenos, } \\
\text { cremas, jaleas, coberturas y derivados del azúcar, como } \\
\text { merengues y coberturas de chocolate. }\end{array}$ \\
\hline $\begin{array}{l}\text { P4. CC5. Identifica los diferentes tipos de masas ideales } \\
\text { para la producción de tortas y pasteles según las bases } \\
\text { de la cocina regional, internacional y de vanguardia. }\end{array}$ & $\begin{array}{l}\text { P4. CF5. Prepara y coordina la elaboración de masas se- } \\
\text { cas y bizcochuelos en general (masas quebradas, masas } \\
\text { de hojaldre, masas para galletas, bizcochuelos básicos, } \\
\text { otros). }\end{array}$ \\
\hline $\begin{array}{l}\text { P4. CC6. Identifica los diferentes tipos de masas saladas } \\
\text { para la producción de productos terminados de calidad } \\
\text { según las bases de la cocina regional, internacional y de } \\
\text { vanguardia. }\end{array}$ & $\begin{array}{l}\text { P4. CF6. Prepara y elabora pastelería de sal (tartas, em- } \\
\text { panadas, pizzas, canapés, bocadillos, otros). }\end{array}$ \\
\hline $\begin{array}{l}\text { P4. CC } 7 \text {. Reconoce las técnicas para decoración y } \\
\text { montaje de tortas de acuerdo con las normas de estética } \\
\text { visual. }\end{array}$ & $\begin{array}{l}\text { P4. CF7. Realiza y coordina la decoración de productos } \\
\text { de pastelería de acuerdo con las políticas del estableci- } \\
\text { miento. }\end{array}$ \\
\hline
\end{tabular}

Fuente: Elaboración propia (2019). 
Tabla 8. Dimensiones de las competencias cognitiva y funcional.

Puesto 5: repostero

\begin{tabular}{|c|c|}
\hline Ocupacionales cognitivas & Ocupacionales funcionales \\
\hline $\begin{array}{l}\text { P5. Cc1. Identifica el contenido de la receta estándar } \\
\text { según las normas de la cocina regional, internacional y } \\
\text { de vanguardia. }\end{array}$ & $\begin{array}{l}\text { P5. CF1. Aplica los procedimientos detallados que } \\
\text { contiene la receta estándar en el momento de proceder } \\
\text { a la transformación de la materia prima y dar paso al } \\
\text { producto final terminado. }\end{array}$ \\
\hline $\begin{array}{l}\text { P5. CC2. Identifica técnicas que se aplican en repostería } \\
\text { de acuerdo con las bases de la cocina regional, interna- } \\
\text { cional y de vanguardia. }\end{array}$ & $\begin{array}{l}\text { P5. CF2. Prepara y coordina la elaboración de masas se- } \\
\text { cas y bizcochuelos en general (masas quebradas, masas } \\
\text { de hojaldre, masas para galletas, bizcochuelos básicos, } \\
\text { entre otros). }\end{array}$ \\
\hline $\begin{array}{l}\text { P5. CC3. Identifica técnicas para decorar productos de } \\
\text { repostería según las normas estéticas, de diseño y la } \\
\text { teoría del uso del color. }\end{array}$ & $\begin{array}{l}\text { P5. CF3. Decora el producto final terminado aplicando } \\
\text { rellenos, baños y coberturas, y empleando las técnicas, } \\
\text { las herramientas y los soportes adecuados. }\end{array}$ \\
\hline $\begin{array}{l}\text { P5. CC4. Identifica los equipos de repostería usados para } \\
\text { la decoración y el montaje de platos según las normas } \\
\text { estéticas, de diseño y la teoría del uso del color. }\end{array}$ & $\begin{array}{l}\text { P5. CF4. Aplica los conocimientos aprendidos para el } \\
\text { uso de los equipos que se utilizan en la decoración de } \\
\text { postres. }\end{array}$ \\
\hline $\begin{array}{l}\text { P5. CC5. Reconoce los principios que se aplican en la } \\
\text { decoración de postres y piezas de repostería para plas- } \\
\text { marlos en el montaje del servicio según las especifica- } \\
\text { ciones de la receta estándar. }\end{array}$ & $\begin{array}{l}\text { P5. CF5. Diseña bocetos en los que aplica las técnicas } \\
\text { para decoración de postres y piezas de repostería. }\end{array}$ \\
\hline $\begin{array}{l}\text { P5. cc6. Reconoce los principios de conservación de } \\
\text { alimentos y su capacidad para manejar tiempos de coc- } \\
\text { ción, temperaturas en las materias primas, productos en } \\
\text { proceso y productos terminados de repostería según las } \\
\text { normas de seguridad e higiene alimentaria, las normas } \\
\text { de calidad o los puntos críticos de control. }\end{array}$ & $\begin{array}{l}\text { P5. CF6. Aplica los principios para conservar postres ter- } \\
\text { minados según su naturaleza para garantizar su estado } \\
\text { e higiene. }\end{array}$ \\
\hline $\begin{array}{l}\text { P5. CC7. Identifica sistemas de calidad en la repostería, } \\
\text { aspectos de supervisión y control de equipos de pastele- } \\
\text { ría, y utensilios que se usan en la elaboración de varie- } \\
\text { dad de productos de acuerdo con las normas de calidad. }\end{array}$ & $\begin{array}{l}\text { P5. CF7. Emplea sistemas de control preventivo y } \\
\text { correctivo en los equipos de pastelería y diversos utensi- } \\
\text { lios para garantizar el óptimo uso de los insumos. }\end{array}$ \\
\hline
\end{tabular}

Fuente: Elaboración propia (2019).

Tabla 9. Dimensiones de las competencias cognitiva y funcional.

Puesto 6: panadero

\begin{tabular}{|l|l|}
\hline \multicolumn{1}{|c|}{ Ocupacionales cognitivas } & \multicolumn{1}{c|}{ Ocupacionales funcionales } \\
\hline $\begin{array}{l}\text { P6. Cc1. Identifica técnicas para la elaboración de } \\
\text { productos de panadería de acuerdo con las bases de la } \\
\text { cocina regional, internacional y de vanguardia. }\end{array}$ & $\begin{array}{l}\text { P6. CF1. Elabora diferentes tipos de masas aplicando } \\
\text { técnicas según la variedad de pan. }\end{array}$ \\
\hline $\begin{array}{l}\text { P6. CC2. Identifica las herramientas y los equipos que } \\
\text { sirven de base en la producción de pan según los están- } \\
\text { dares que rigen en la industria. }\end{array}$ & $\begin{array}{l}\text { P6. CF2. Utiliza herramientas tecnológicas que le permi- } \\
\text { tan estandarizar los procesos en la producción de pan. }\end{array}$ \\
\hline $\begin{array}{l}\text { P6. CC3. Identifica el contenido de la receta estándar } \\
\text { según las normas de panadería regional, internacional y y } \\
\text { de vanguardia. }\end{array}$ & $\begin{array}{l}\text { P6. CF3. Aplica los procedimientos detallados que } \\
\text { contiene la receta estándar en el momento de proceder } \\
\text { a la transformación de la materia prima y dar paso al } \\
\text { producto final terminado. }\end{array}$ \\
\hline
\end{tabular}


Ocupacionales cognitivas

P6. Cc4. Conoce de sistemas, métodos y documentos que se aplican para la gestión de inventarios de acuerdo con los sistemas de rotación, como FIFO, LIFO Y PROMEDIO.

P6. Cc5. Identifica los procesos en la elaboración del pan y técnicas de decoración de productos de acuerdo con las bases de la cocina regional, internacional y de vanguardia.

P6. cc6. Identifica los principios básicos de compra de productos según las normas de calidad requeridas.

P6. CC7. Reconoce los procesos de almacenamiento, conservación y rotación de materias primas según las normas de seguridad e higiene alimentaria y las normas de calidad propuestas para tal efecto.
Ocupacionales funcionales

P6. CF4. Emplea documentos de control en la bodega con el interés de controlar los desperdicios.

P6. CF5. Lleva a cabo las actividades que deben desarrollarse en el proceso para elaborar pan y decorar productos.

P6. CF6. Aplica los principios básicos que debe tener la materia prima de acuerdo con los requerimientos planteados en el control de la calidad del producto.

P6. CF7. Usa técnicas correctas para la gestión y conservación de materias primas.

Fuente: Elaboración propia (2019).

Tabla 10. Dimensiones de las competencias cognitiva y funcional.

Puesto 7: mesero

\begin{tabular}{|c|c|}
\hline Ocupacionales cognitivas & Ocupacionales funcionales \\
\hline $\begin{array}{l}\text { P7. CC1. Identifica las operaciones de marcado y el des- } \\
\text { barazado de las mesas según el protocolo establecido. }\end{array}$ & $\begin{array}{l}\text { P7. CF1. Aplica los protocolos correspondientes para } \\
\text { preparar la mesa de acuerdo con el tipo de servicio. }\end{array}$ \\
\hline $\begin{array}{l}\text { P7. CC2. Reconoce las técnicas, los métodos y la cultura } \\
\text { general de acuerdo con la oferta gastronómica del esta- } \\
\text { blecimiento. }\end{array}$ & $\begin{array}{l}\text { P7. CF2. Aplica conocimientos de bases de cocina con } \\
\text { la finalidad de asesorar adecuadamente al cliente al mo- } \\
\text { mento de tomar su orden. }\end{array}$ \\
\hline $\begin{array}{l}\text { P7. CC3. Reconoce el protocolo en restauración de } \\
\text { acuerdo con la ubicación de los comensales y el flujo } \\
\text { del servicio. }\end{array}$ & $\begin{array}{l}\text { P7. CF3. Aplica el correcto desempeño de funciones del } \\
\text { servicio. }\end{array}$ \\
\hline $\begin{array}{l}\text { P7. CC4. Define las técnicas de ventas para una correcta } \\
\text { atención al cliente según las normas de mercadeo, las } \\
\text { técnicas de venta y el protocolo de servicio. }\end{array}$ & $\begin{array}{l}\text { P7. CF } 4 \text {. Resuelve adecuadamente los requerimientos } \\
\text { del cliente y lo asesora en su orden. }\end{array}$ \\
\hline $\begin{array}{l}\text { P7. CC5. Identifica los requerimientos y las necesidades } \\
\text { de los clientes de acuerdo con los principios de la comu- } \\
\text { nicación efectiva. }\end{array}$ & $\begin{array}{l}\text { P7. CF5. Resuelve inquietudes sobre preparaciones, } \\
\text { ingredientes y métodos de cocción de las distintas pre- } \\
\text { paraciones. }\end{array}$ \\
\hline $\begin{array}{l}\text { P7. CC6. Identifica el protocolo que debe aplicarse en } \\
\text { la entrega de las preparaciones gastronómicas ante el } \\
\text { cliente y el debido uso de la cubertería según las normas } \\
\text { de protocolo. }\end{array}$ & $\begin{array}{l}\text { P7. CF6. Aplica conocimientos para la entrega de las } \\
\text { órdenes al cliente de acuerdo con el origen de la prepa- } \\
\text { ración. }\end{array}$ \\
\hline $\begin{array}{l}\text { P7. CC7. Reconoce los problemas en el servicio y la } \\
\text { forma de comportarse frente al cliente de acuerdo con } \\
\text { los procedimientos establecidos para la resolución de } \\
\text { conflictos. }\end{array}$ & $\begin{array}{l}\text { P7. CF7. Aplica técnicas de seguimiento a las necesida- } \\
\text { des varias de los clientes o a los conflictos que puedan } \\
\text { presentarse en el momento del servicio. }\end{array}$ \\
\hline
\end{tabular}

Fuente: Elaboración propia (2019). 


\section{Competencias personales}

El primer estudio empírico relacionado con las competencias en el ámbito de la hotelería fue publicado por Richard Tas (1988) en un artículo que presenta la investigación realizada en grandes hoteles de EE. UU., y en el que se compilan 36 competencias necesarias para el gerente de hotel. Las competencias fueron calificadas por medio de la escala de Likert, de 1 a 5 , donde, en promedio, la puntuación más baja fue de 2,5, lo que, según el autor, enuncia que todos los atributos eran de gran importancia. El estudio revela que, de las 36 competencias, 6 atributos relacionados con las competencias personales son considerados esenciales, 18 fueron estimados como de importancia considerable y 12 tienen una moderada relevancia.

Por su parte, Gersh (2016) realizó un estudio respecto a las percepciones de practicantes y educadores de la industria culinaria. En su trabajo, el autor clasificó 40 declaraciones de competencias en 5 dimensiones de competencia: 13 administrativas, 7 de liderazgo, 8 interpersonales, 7 conceptuales y 5 técnicas. Luego de unificar los criterios de educadores y practicantes - es decir, de quienes se inician en la industria-, concluyó que ambos grupos califican las competencias que forman parte del constructo interpersonal como competencias básicas esenciales; por lo tanto, las más importantes son, en su orden, la competencia técnica, la de liderazgo, la administrativa y la conceptual.

Por considerar las competencias personales sociales como esenciales para la gestión del talento humano, se realiza una descripción de estas competencias, que también en el presente estudio fueron consideradas como imprescindibles; se exponen en grado de importancia de mayor a menor:

1. Compromiso con la organización. Capacidad para hacer de los objetivos de la empresa parte de sus objetivos personales y que estos últimos no estén sobre los de la organización. Significa adherirse a los principios éticos de la empresa, a su misión y visión.

2. Ética. Capacidad para dirigir y controlar al equipo de trabajo. Demostrar seguridad al desarrollar las funciones encomendadas. Implica el desempeño correcto dentro de las organizaciones, hacer uso de buenas costumbres, de valores éticos y morales.

3. Flexibilidad y adaptación. Profesional con un buen manejo de relaciones humanas, dispuesto a trabajar en cualquier área y que comprende las necesidades de la empresa.

4. Innovación y creatividad. Facultad para solucionar problemas relacionados con el puesto de trabajo o la actividad a cargo. Ser capaz de crear alternativas en la transformación del producto y del servicio, para lo cual se ajusta a las necesidades del mercado y genera valor agregado diferenciador en comparación con la competencia.

5. Integridad. Procedimiento conductual con base en la formación ética del profesional, con la finalidad de crear un buen ambiente laboral entre sus compañeros y áreas de trabajo, basado en los valores propios y los de la empresa.

6. Prudencia. Aptitud para diseñar y hacer cumplir normas institucionales en beneficio de la organización y sus asociados, de modo que se logre alcanzar los objetivos trazados por la empresa. Es la capacidad de discernir los buenos y malos momentos de la organización, de los clientes, los proveedores y demás clientes con los que se relaciona. 
7. Respeto. Capacidad para identificarse y alinear la conducta personal con los valores institucionales; acepta, respeta y promueve en su ámbito laboral los valores y objetivos de la empresa, y actúa en consecuencia con los valores de esta; trata con respeto e igualdad a todas las personas. (Universidad de Santiago de Chile, 2013).

8. Responsabilidad. Capacidad para llevar a cabo las funciones y responsabilidades inherentes al puesto de trabajo bajo estándares de calidad; busca la mejora continua y propone la adaptación y modernización de los procesos (Universidad de Santiago de Chile, 2013).

9. Temple. Aptitud para enfrentar los retos del ámbito laboral con serenidad y valentía, manteniendo la calma ante las situaciones de estrés propias de la profesión.

10. Disciplina. Capacidad para seguir las reglas de comportamiento para mantener el orden y la subordinación entre los miembros del equipo de trabajo (Alles, 2015b).

\section{Conclusiones}

Las empresas de servicios, específicamente las de restauración, a nivel local y mundial se enfrentan a la necesidad de instaurar competencias laborales clave basadas en la aplicación y combinación de conocimiento, habilidades, actitudes positivas y valores que permitan desarrollar de forma exitosa la actividad profesional.

Por lo general, las empresas y sus colaboradores tratan continuamente de mejorar su desempeño laboral, lo que implica un cambio efectivo del comportamiento en el trabajo, aprendizaje y desaprendizaje de conocimientos, así como el desarrollo de otras habilidades. Si se toman en cuenta estos elementos, el rendimiento depende no solo de estrategias, estructura, sistemas y operatividad, sino del accionar del stock de recursos que posee una organización y de la exploración de otros que el entorno demanda.

Los miembros de la organización realizan actividades laborales no solo para satisfacer sus aspiraciones personales (remuneración, desarrollo y progreso), sino, además, para contribuir a los objetivos de la organización; de no ser así, ninguno de ellos tiene justificativo válido.

Los elementos que desempeñan un papel importante en el proceso de cambio de comportamiento en el trabajo son:

1. La gestión de competencias laborales. Estas competencias requieren de cambios de comportamiento y mejoras constantes dentro y fuera del trabajo.

2. Los actores. Es decir, los recursos humanos son quienes, basados en sus competencias y motivación, se apropian de actividades para provocar el desarrollo, el rendimiento y la eficiencia organizacional.

3. Las intervenciones son las acciones del individuo orientadas a conseguir un cambio organizacional. Estas acciones son producto de los procesos de capacitación, desarrollo cognitivo y habilidades específicas de puestos de trabajo; las intervenciones además deben incluir procesos de transferencia de información y conocimiento, espacios donde juegan un papel importante las competencias personales sociales y las metacompetencias.

4. El contexto del proceso. Se requiere una estructura organizacional más orgánica, que enfrente los retos del entorno interno 
y externo de manera más participativa, con recursos humanos y características personales en constante evolución, que motiven el desarrollo personal y empresarial.

Por lo expuesto, las competencias aquí estudiadas están formadas por dos dimensiones. La primera está compuesta por la dimensión de las competencias conceptuales - es decir, aquellos conocimientos propios de la disciplina de los servicios-y las competencias operacionales — las habilidades funcionales técnicas predictoras de un buen desempeño laboral-.

La segunda dimensión está conformada por las competencias personales, integradas por las metacompetencias y las competencias sociales. En concordancia con los objetivos, los resultados y el marco de competencias propuesto, se han determinado alrededor de 50 competencias conceptuales, 50 operacionales y 10 personales.

Los resultados de la investigación resaltan como imprescindibles y más importantes las competencias personales en comparación con las ocupacionales. En orden de importancia, se destacan las siguientes: compromiso con la organización, ética, flexibilidad y adaptación, innovación y creatividad, integridad, prudencia, respeto, responsabilidad, temple y disciplina. Esta información es coherente con otros estudios realizados en el área de la hotelería en los que los atributos relacionados con las competencias personales han sido calificados con escalas de esenciales, importantes y de moderada importancia. Así mismo, otros trabajos empíricos llevados a cabo en el área de servicios han identificado que educadores y practicantes califican el constructo interpersonal como competencias básicas esenciales y, por lo tanto, el más relevante. Una limitante en esta investigación fue no incluir las metacompetencias como una variable.
A manera de conclusión, se puede enunciar que un sistema basado en competencias laborales debe contar con tres criterios básicos:

1. Sistema de normalización. Lo que una persona debe ser capaz de hacer, que pueda demostrar y evidenciarse. Este sistema debe estar normalizado.

2. Sistema de evaluación y certificación de competencias. El proceso de evaluación de competencias debe permitir la certificación de las competencias de la persona o del trabajador; esta evaluación la debe hacer un organismo acreditado y especializado. Como se ha evidenciado en los estudios, estos organismos reguladores de la evaluación y certificación deben orientar sus procesos de formación hacia sistemas más holísticos, porque las empresas valoran las competencias personales sobre las ocupacionales.

3. Formación basada en competencias. Contar con un sistema de formación que garantice una formación holística de calidad y la mejora de la empleabilidad. Es en esta instancia en la que organismos del Estado, empresas e instituciones de formación deben orientar su enfoque hacia macro, meso y microcurrículos, así como a sistemas de evaluación de desempeño laboral capaces de dar respuesta a los problemas de la sociedad y sus contextos.

\section{Implicaciones teóricas}

Tanto los trabajos académicos como los procesos de enseñanza-aprendizaje que están basados en enfoques de competencias tienden a potenciar la dimensión ocupacional, es decir, se orientan a desarrollar las competencias en los niveles cognitivo y funcional. Este estudio, basado en la información de las entrevistas y en la revisión de puestos de trabajo clave en empresas de restauración, 
propone un marco de competencias para siete puestos clave, con un promedio de 100 competencias y sus definiciones en tres dimensiones: cognitiva, funcional y social.

En un mundo globalizado, competitivo y cambiante, la adquisición de competencias en todas sus dimensiones es necesaria para hacer del recurso humano un intangible de alto valor, capaz de proporcionar ventaja competitiva de mercado.

\section{Implicaciones prácticas}

Este marco de competencias puede ser usado en dos vías: por un lado, las instituciones de educación de nivel técnico podrían usarlo para orientar sus planes curriculares de formación, considerando que los empresarios valoran en mayor medida la dimensión de competencias personales (sociales) sobre las ocupacionales.

Por el lado del empresario, podría servir para guiar sus sistemas de gestión de recursos humanos: reclutamiento, selección, análisis de puestos, evaluación, formación y desarrollo de talentos. Así mismo, serviría de guía para el diseño y rediseño de puestos de trabajo, de esta forma se orientarían hacia planes de capacitación formal e informal, transferencia de conocimientos tácitos entre trabajadores y fortalecimiento de conocimientos explícitos entre los miembros de una empresa.

Por último, se considera que este trabajo contribuye a aclarar cuáles son las competencias en los ámbitos ocupacional y personal que el talento humano que labora en la industria restaurantera debe desarrollar en su entorno profesional, personal y organizacional.

\section{Referencias bibliográficas}

Alles, M. (2015a). Diccionario de competencias: la trilogía - Vol. 1. Las 60 competencias más utilizadas en gestión por competencias (2. ${ }^{\mathrm{a}} \mathrm{ed}$.). Buenos Aires: Ediciones Granica.

Alles, M. (2015b). Dirección estratégica de recursos humanos: gestión por competencias ( $3 .^{\mathrm{a}} \mathrm{ed}$.). Buenos Aires: Ediciones Granica.

Arroyo, R. (2012). Habilidades gerenciales: desarrollo de destrezas, competencias y actitud. Bogotá: ECOE Ediciones.

Baradarani, S. y Kilic, H. (2017). Service innovation in the hotel industry: Culture, behavior, performance. The Service Industries Journal, 38(13-14), 897-924. DOI: 10.1080/02642069.2017.1420172

Chiavenato, I. (2009). Gestión de talento humano. México, D. F.: McGraw-Hill Interamericana.

Echeverría, B. (2002). Gestión de la competencia de acción profesional. Revista de Investigación Educativa, 20(1), 7-43. Recuperado de http:// revistas.um.es/rie/article/view/97411/93521

Gersh, I. (2016). Culinary industry practitioners' and educators' perceptions of core competencies for a 4-year Bachelor's degree in the culinary arts. Journal of Hospitality \& Tourism Education, 28(1), 32-43. DOI: 10.1080/10963758.2015.1127166

Gutiérrez, E. (2010). Competencias gerenciales: habilidades, conocimientos, aptitudes. Bogotá: ECOE Ediciones.

Instituto Nacional de Cualificaciones. (2018). Hotelería y turismo. Recuperado de http://incual. mecd.es/hosteleria_cualificaciones

Instituto Nacional de Estadística y Censos (INEC). (2014). Encuesta de hoteles. Restaurantes y servicios-2014. Recuperada de https://www.ecuadorencifras.gob.ec/ hoteles-restaurantes-y-servicios-2014/

Lazzati, S. (2008). El cambio del comportamiento en el trabajo. Buenos Aires: Ediciones Granica.

Rev. Turismo y Sociedad, vol. xxVII, julio-diciembre 2020, pp. 161-181 
Le Deist, F. D. y Winterton, J. (2005). What is competence? Human Resource Development International, 8(1), 27-46. DOI: $10.1080 / 1367886042000338227$

Lepsinger, R. y De Lucía, A. (1999). The art and science of competency models. San Francisco: Jossey-Bass Publishers.

Ministerio de Turismo. (2017). Boletín de Estadisticas Turísticas. Recuperado de https://servicios. turismo.gob.ec/turismo-cifras

Ministerio de Turismo. (2019). Turismo en cifras. Recuperado de https://servicios.turismo.gob.ec/ turismo-cifras

Morales, G. (2011). Currículo por competencias. Guayaquil: Ediciones de la U. de Guayaquil.

Nonaka, I. (1994). A dynamic theory of organizational knowledge creation. Organization Science, 5(1), 14-37. DOI: 10.1287/orsc.5.1.14

Organización Internacional del Trabajo (OIT). (4 de septiembre de 2012). ¿Qué es competencia? Recuperado de http://www. oitcinterfor.org/p \% C3\% A 1 gina-libro/ 1 $\% \mathrm{C} 2 \% \mathrm{BFqu} \% \mathrm{C} 3 \% \mathrm{~A} 9$-competencia-laboral

Romero, I. L. (2006). Las pyme en la economía global. Hacia una estrategia de fomento empresarial. Problemas del Desarrollo, 37(146), 31-50. Recuperado de http://www.scielo.org.mx/pdf/ prode/v37n146/v37n146a3.pdf

Schkolnik, M., Araos, C. y Machado, F. (2005). Certificación por competencias como parte del sistema de protección social: la experiencia de países desarrollados y lineamientos para América Latina (Serie Políticas Sociales, n. ${ }^{0}$ 113). Santiago de Chile: CEPAL.

Secretaría de Educación Pública (SEP). (2018). Competencias de personas y perfiles ocupacionales. México: SEP. Recuperado de https:// conocer.gob.mx/wp-content/uploads/2017/05/ MonografiasPerfilesOcupacionalesTotales.pdf

Secretaría Técnica del Sistema Nacional de Cualificaciones Profesionales (SETEC) Ecuador. (2017). Informe de Rendición de Cuentas. Quito: SETEC Ecuador. Recuperado de http://www.cualificaciones.gob.ec/wp-content/uploads/2018/03/InformeRendicion-de-Cuentas-2017.compressed.pdf

Secretaría Técnica del Sistema Nacional de Cualificaciones Profesionales (SETEC) Ecuador. (2018). Catálogo nacional de cualificaciones. Quito: SETEC Ecuador.

Servicio Nacional de Aprendizaje (SENA). (2011). Evaluación y certificación por competencias laborales. Recuperado de http://www.sena.edu. co/es-co/formacion/Paginas/Evaluaci\%C3\%B3ny-Certificaci $\% \mathrm{C} 3 \% \mathrm{~B} 3 \mathrm{n}$-por-competenciaslaborales.aspx

Tas, R. F. (1988). Teaching future managers. Cornell Hotel and Restaurant Administration Quarterly, 29(2), 41-43. DOI: 10.1177/001088048802900215

Universidad de Santiago de Chile. (2013). Diccionario de competencias. Santiago de Chile: Universidad de Santiago de Chile. Recuperado de http://ddp.usach.cl/sites/ddp/files/documentos/ diccionario_de_competencias_0.pdf

Yance, C., Solís, L., Burgos, I. y Hermida, L. (2017). La importancia de las pymes en el Ecuador. Revista Observatorio de la Economía Latinoamericana [En línea]. Recuperado de http://www.eumed.net/cursecon/ecolat/ec/2017/ pymes-ecuador.html

Yap, M. H. T., Jung, T. H. y Kisseleff, J. (2015). Educators' perspectives of eLearning in Swiss private hospitality institutions. Journal of Hospitality \& Tourism Education, 27(4), 180-187. DOI: 10.1080/10963758.2015.1089509 
